\title{
Sequencing Therapy for Patients With Lung Cancer
}

Presented by Gregory J. Riely, MD, PhD

\section{ABSTRACT}

Non-small cell lung cancer (NSCLC) can no longer be considered as one disease, nor can it be treated as one. Understanding tumor histology in NSCLC is critical to understanding optimal biomarker evaluation and initial therapy. Proper biomarker evaluation includes both evaluation of PD-L1 status, as well as testing for actionable oncogenic drivers such as EGFR, ALK, ROS1, BRAF, Met Exon 14, RET, and NTRK. For patients with NSCLC and a driver oncogene, preferred treatment is targeted therapy. Conversely, for those without an oncogenic driver, preferred initial treatment is pembrolizumab in combination with chemotherapy for patients with low PD-L1 expression (1\%-49\%) or as a single-agent for high PD-L1 expression ( $\geq 50 \%$ ). For small cell lung cancer (SCLC), the first major NCCN Guideline changes occurred in 2019, with the addition of either atezolizumab or durvalumab to platinum-based chemotherapy and etoposide as first-line therapy for patients with extensive-stage SCLC.

J Natl Compr Canc Netw 2020;18(7.5):945-948 doi: $10.6004 /$ jnccn.2020.5010

Outcomes in non-small cell lung cancer (NSCLC) are improving in part to the integration of molecular analysis and PD-L1 testing into standard practice. Classifying and treating these malignancies according to their oncogenic driver mutations and level of PD-Ll expression informs initial therapy for patients, which in turn translates to more tailored treatments and better outcomes, according to Gregory J. Riely, MD, PhD, Vice Chair of Clinical Research, Department of Medicine, Memorial Sloan Kettering Cancer Center. The first step to determining initial therapy for lung cancer is histology. "This is what we've been doing for 50 years, and it still guides our approach to treatment," he said at the NCCN 2020 Virtual Annual Conference. "One of the reasons we use histology is to help determine which patients need which biomarkers evaluated," he said.

The NCCN Clinical Practice Guidelines in Oncology (NCCN Guidelines) for NSCLC recommend molecular testing for all patients with metastatic adenocarcinoma, and state that testing should be considered for certain patients with metastatic squamous cell carcinoma. ${ }^{1}$ Factors used to identify the need for molecular testing in patients with metastatic squamous cell carcinoma are described in Figure 1. In order to test for all of these molecular targets, the NCCN Guidelines strongly advise broad molecular profiling because large panel testing is the most efficient approach to obtaining all of the necessary information from the available tissue.

NSCLC is classified according to mutations and gene fusions. The guidelines highlight some key molecular subtypes that should be included in molecular testing:
EGFR sensitizing mutations, $A L K$ gene rearrangements, $R O S 1$ rearrangements, $B R A F$ mutations, $R E T$ rearrangements, mutations that lead to MET exon 14 skipping, and $N T R K$ fusions. Identifying these molecular targets informs the initial course of treatment for patients with NSCLC. Beyond molecular testing, all patients (regardless of histology) should have PD-L1 testing (negative, low, and high). "The way PD-L1 testing is done is a bit of a moving target right now, but I would emphasize that it has to be performed for all patients with NSCLC," said Dr. Riely. "Testing results lead to different pages of the NCCN Guidelines, which in turn guides therapy for individual patients," he said.

For the first-line treatment of patients with advanced NSCLC, the information collected through histologic assessment, molecular analysis, and PD-L1 testing leads treatment to 1 of 3 paths: patients with identified gene mutations or rearrangements comprise approximately $25 \%$ of patients, those with low or no PD-L1 make up approximately $45 \%$, and those with high PD-L1 expression $(\geq 50 \%$ ) represent another $30 \%$ of patients, said Dr. Riely.

\section{Treating EGFR-Mutated NSCLC}

"EGFR-sensitizing mutations are the molecular subtype we've known about and have been treating the longest," he said. Multiple randomized trials have shown that EGFR tyrosine kinase inhibitors (TKIs) are better than platinumbased doublets for patients with EGFR-mutant (positive) metastatic NSCLC, and the data for gefitinib, erlotinib and afatinib have all demonstrated this benefit. ${ }^{2-5}$ 


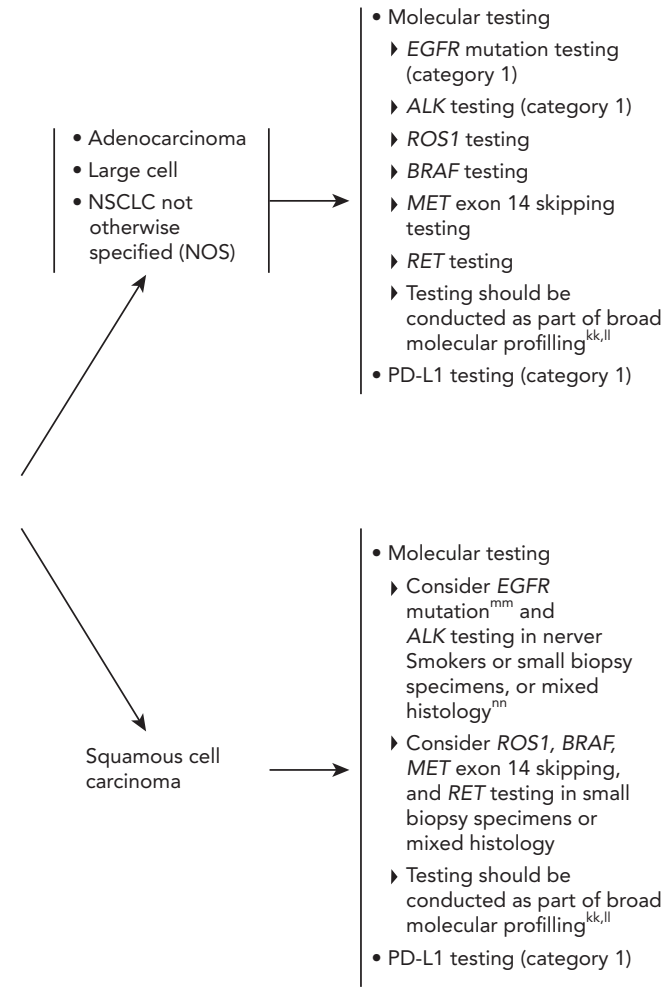

Figure 1. After histology, then biomarker evaluation. From the Ettinger DS, Wood DE, Aisner DL, et al. NCCN Clinical Practice Guidelines in Oncology: Non-Small Cell Lung Cancer. Version 4.2020. Accessed April 1, 2020. Available at: NCCN.org

According to Dr. Riely, all patients will develop acquired resistance to these EGFR TKIs. But a second mutation, EGFR T790M, has been identified in approximately $66 \%$ of patients at the time of acquired resistance, which can be targeted with the third-generation EGFR TKI osimertinib. "Osimertinib is the newest drug in this arena," he noted. "It targets both EGFR L858R exon 19 deletions, EGFR exon 29 deletions, and EGFR T790M, and importantly, it's relatively sparing of wild-type EGFR." In clinical trials, osimertinib has demonstrated superiority over platinum-based chemotherapy in the presence of EGFR T790M mutations in the context of resistance. ${ }^{7}$

Not long ago, the treatment paradigm for patients with EGFR-mutant metastatic NSCLC included a firstgeneration TKI in the frontline setting, followed by osimertinib for patients with T790M mutations or chemotherapy for those without T790M mutations. This led to the question of whether osimertinib might be effective in the first-line, and a clinical trial demonstrated a significant progression-free survival (PFS) benefit for osimertinib compared with gefitinib/erlotinib as first-line treatment for advanced NSCLC (18.9 vs 10.2 months; $P<.001){ }^{8}$ The trial also showed an overall survival (OS) benefit in patients on osimertinib. "I think the truly impressive thing was seeing a median OS for patients with EGFR-mutant lung cancer that exceeds 3 years," he noted. "That's a dramatic improvement from what we once saw, but clearly shows us that we have a long way to go."

Based on these data, osimertinib was added to the NCCN Guidelines for NSCLC as the preferred first-line treatment for patients with sensitizing EGFR mutations and metastatic NSCLC. According to Dr. Riely, more recent data suggest that erlotinib + ramucirumab may lead to similar PFS compared with osimertinib, ${ }^{10}$ although this drug combination is still only listed as "other recommended" in the current guidelines.

\section{Treating ALK-Positive Metastatic NSCLC}

Crizotinib has previously demonstrated longer PFS than cisplatin/pemetrexed in the first-line treatment of patients with ALK-positive NSCLC. "1 "But while crizotinib was showing its value, second-generation ALK inhibitors were being developed such as alectinib, ceritinib, and brigatinib," he said. These drugs are more selective for ALK, but don't have a notable effect on MET (the original target of crizotinib). Additionally, they can inhibit mutated ALK (a mechanism of resistance), and importantly, they may have greater activity in the central nervous system, he added.

Initial clinical data for alectinib in patients with ALKpositive metastatic NSCLC previously treated with and have developed resistance to crizotinib showed "striking benefits" in patients on alectinib. ${ }^{22}$ "There are similar data for both ceritinib and brigatinib, suggesting that these second-generation ALK inhibitors all had the ability to overcome resistance to crizotinib," he said. This led to a similar question to the one posed for patients with EGFR-mutated tumors: should patients with ALKpositive metastatic NSCLC be started on secondgeneration ALK inhibitors?

Data from clinical trials comparing second-generation ALK inhibitors to crizotinib showed a clear survival benefit for the newer ALK inhibitors. ${ }^{13,14}$ In patients with $A L K$ rearrangements discovered before first-line systemic therapy, the preferred treatment outlined in the current NCCN Guidelines is now alectinib; other recommended treatments include brigatinib and ceritinib. "I think these decisions around preference were related to toxicities observed," noted Dr. Riely.

Available drugs and preferred first-line treatments for the 7 actionable molecular subtypes of NSCLC highlighted in the NCCN Guidelines are listed in Figure 2.

\section{Tailoring Treatment to PD-L1 Expression}

According to Dr. Riely, for the majority of patients with metastatic NSCLC, decision-making in regard to first-line treatment is focused around PD-L1 status. In patients 


\begin{tabular}{|c|c|c|c|}
\hline Target & \multicolumn{2}{|c|}{ Available } & Preferred First Line \\
\hline EGFR & $\begin{array}{c}\text { Gefitinib } \\
\text { Afatinib } \\
\text { Dacomitinib }\end{array}$ & $\begin{array}{c}\text { Erlotinib } \\
\text { Osimertinib }\end{array}$ & Osimertinib \\
\hline ALK fusions & $\begin{array}{c}\text { Crizotinib } \\
\text { Alectinib }\end{array}$ & $\begin{array}{c}\text { Ceritinib } \\
\text { Brigatinib }\end{array}$ & Alectinib \\
\hline BRAF V600E & Dabrafenib + trametinib & Dabrafenib + trametinib \\
\hline ROS, fusions & $\begin{array}{c}\text { Crizotinib } \\
\text { Ceritinib }\end{array}$ & $\begin{array}{c}\text { Entrectinib } \\
\text { Lorlatinib }\end{array}$ & Crizotinib, entrectinib \\
\hline NTRK fusions & \multicolumn{2}{|c|}{ Larotrectinib, entrectinib } & Larotrectinib, entrectinib \\
\hline RET fusions & \multicolumn{2}{|c|}{$\begin{array}{c}\text { Selpercatinib, cabozantinib, } \\
\text { vandetanib }\end{array}$} & Selpercatinib \\
\hline MET exon 14 & \multicolumn{2}{|c|}{ Capmatinib, crizotinib } & Capmatinib \\
\hline
\end{tabular}

Figure 2. NSCLC is no longer one disease.

with high PD-L1 expression ( $\geq 50 \%$ ), the PD-1 inhibitor pembrolizumab previously demonstrated a dramatic improvement in PFS compared with chemotherapy. ${ }^{15}$ Updated OS data from this trial has shown a significantly improved median OS versus chemotherapy. ${ }^{16}$ "Even though there was crossover allowed, the median OS [with pembrolizumab] is now approaching 2.5 years," he said.

Although pembrolizumab and other immune checkpoint inhibitors are associated with fewer adverse events (AEs) than chemotherapy, specific immune-related AEs are associated with these treatments, including reactions like hypo/hyperthyroidism, which can usually be easily managed, but also more serious side effects such as severe skin reactions, colitis, nephritis, and Type 1 diabetes. "These are important AEs to know about so clinicians can anticipate management in patients who received pembrolizumab," said Dr. Riely.

Frontline data for single-agent checkpoint inhibitor therapy show that pembrolizumab is a better option than chemotherapy in patients with metastatic NSCLC whose tumor staining shows PD-L1 expression $\geq 50 \%$. However, for those with PD-L1 expression of $1 \%$ to $49 \%$, pembrolizumab has not demonstrated superiority over chemotherapy. ${ }^{17}$ "As we dilute out patients with high PD-L1 expression, we see less and less of a signal," he said.

Similar trials have been conducted in the first-line setting comparing nivolumab and durvalumab to chemotherapy and using different PD-L1 cut points (5\% and $25 \%$ ), but they have produced negative results. However, more recent data support the use of single-agent atezolizumab using a novel PD-Ll assay. "This may make it into the guidelines in the future," he added. [Editor's Note: Atezolizumab monotherapy was added to the NCCN Guidelines for NSCLC during the version 5.2020 update.]

Relatively little data exist to guide treatment in patients with both high PD-L1 expression and oncogenic drivers, because most trials that have enrolled patients with high PD-L1 expression excluded those with oncogenic drivers. However, a small phase II study of pembrolizumab in patients with EGFR-mutant, PD-L1-positive, TKI-naïve tumors and advanced NSCLC showed no benefit for pembrolizumab over chemotherapy. ${ }^{18}$ According to Dr. Riely, these findings underscore the importance of using a targeted therapy approach in patients with targetable oncogenic drivers.

The NCCN Guidelines for NSCLC prioritize molecular drivers over PD-L1 status. "It's only in those patients without oncogenic drivers that we think about the value of PD-L1 staining," he reiterated. According to the NCCN Guidelines for NSCLC, patients with metastatic disease and high PD-L1 expression ( $\geq 50 \%$ ) who lack a driver oncogene have preferred options of singleagent pembrolizumab or combination pembrolizumab + chemotherapy. Other treatment options are outlined in the NCCN Guidelines.

\section{Treating Patients With Low PD-L1 Expression}

Patients with metastatic NSCLC and lower PD-L1 staining $(<50 \%)$ are typically recommended for initial treatment with chemotherapy + anti-PD-1/PD-L1 therapy if they have no contraindications to this therapy. In patients with newly diagnosed metastatic non-squamous NSCLC without EGFR or $A L K$ mutations, an influential trial demonstrated that the addition of pembrolizumab to standard chemotherapy resulted in significantly longer OS and PFS compared with chemotherapy alone. ${ }^{19}$ "Importantly, the benefits observed in this trial were seen even in patients with PD-L1-negative expression," he said. "In the subgroup where we'd think the addition of pembrolizumab would have the least benefit ( $\mathrm{PD}-\mathrm{Ll}<1 \%$ ), the hazard ratio for OS was 0.59."

Pembrolizumab is not the only immunotherapy drug to demonstrate value when given in combination with chemotherapy. The addition of atezolizumab to carboplatin/ paclitaxel/bevacizumab also demonstrated a significant survival benefit in patients with nonsquamous NSCLC, regardless of PD-L1 expression and EGFR or $A L K$ genetic alteration status. ${ }^{20}$ The chemo-immunotherapy data for squamous cell lung cancer are similar, and have shown PFS and OS improvements with the addition of pembrolizumab to carboplatin and a taxane, ${ }^{21}$ and a PFS benefit only for atezolizumab when added to carboplatin and a taxane. ${ }^{22}$

\section{Combining Immune Checkpoint Inhibitors}

The combination of ipilimumab and nivolumab improved OS compared with chemotherapy alone in patients with metastatic or recurrent NSCLC who had no prior anticancer therapy, regardless of their PD-L1 status. ${ }^{23}$ Based on the results of this trial, on May 26, 2020, the FDA approved the combination of nivolumab + ipilimumab + chemotherapy 
for the first-line treatment of patients with metastatic NSCLC regardless of tumor PD-Ll expression.

"Importantly, the control arm in this trial was chemotherapy alone," noted Dr. Riely. "It was not what I think many of us would consider the control arm of today, which would have been chemotherapy + pembrolizumab or chemotherapy + bevacizumab/atezolizumab." Dr. Riely noted an important caveat to this study: less chemotherapy does not mean less toxicity. "One of the reasons people wanted to explore the value of a chemotherapyfree regimen was the potential for less toxicity, but serious treatment-related AEs in the nivolumab/ ipilimumab group were actually significantly higher," he said. "So although there's no chemotherapy in the nivolumab/ipilimumab arm, it has a significant toxicity profile and is not to be underestimated."

For SCLC, he added that the first major guideline changes occurred in the past year, with the addition of either atezolizumab or durvalumab to platinum-based chemotherapy and etoposide for the first-line treatment of extensive-stage SCLC. ${ }^{24}$

Disclosures: Dr. Riely has disclosed that the has received grant/research support from Merck \& Co., Inc.; Novartis Pharmaceuticals Corporation; Mirat Therapeutics Inc.; Pfizer Inc.; Roche Laboratories, Inc.; and Takeda Pharmaceuticals North America, Inc.

Correspondence: Gregory J. Riely, MD, PhD, Memorial Sloan Kettering Cancer Center, 530 East 74th Street, New York, NY 10021.

Email: rielyg@mskcc.org

\section{References}

1. Ettinger DS, Wood DE, Aisner DL, et al. NCCN Clinical Practice Guidelines in Oncology: Non-Small Cell Lung Cancer. Version 4.2020. Accessed April 1, 2020. Available at: NCCN.org

2. Mok TS, Wu Y, Thongprasert S, et al. Gefitinib or carboplatin-paclitaxel in pulmonary adenocarcinoma. N Engl J Med 2009;361:947-957.

3. Maemondo M, Inoue A, Kobayashi K, et al. Gefitinib or chemotherapy for non-small-cell lung cancer with mutated EGFR. N Engl J Med 2010;362: 2380-2388.

4. Mitsudomi T, Morita S, Yatabe Y, et al. Gefitinib versus cisplatin plus docetaxel in patients with non-small-cell lung cancer harbouring mutations of the epidermal growth factor receptor (WJTOG3405): an open label, randomised phase 3 trial. Lancet Oncol 2010;11:121-128.

5. Rosell R, Carcereny E, Gervais R, et al. Erlotinib versus standard chemotherapy as first-line treatment for European patients with advanced EGFR mutation-positive non-small-cell lung cancer (EURTAC): a multicentre, openlabel, randomised phase 3 trial. Lancet Oncol 2012;13:239-246.

6. Yu HA, Arcila ME, Rekhtman N, et al. Analysis of tumor specimens at the time of acquired resistance to EGFR-TKI therapy in 155 patients with EGFR-mutant lung cancers. Clin Cancer Res 2013;19:2240-2247.

7. Mok TS, Wu Y, Ahn M, et al. Osimertinib or platinum-pemetrexed in EGFR T790M-positive lung cancer. N Engl J Med 2017;376:629-640.

8. Soria J, Ohe Y, Vansteenkiste J, et al. Osimertinib in untreated EGFR-mutated advanced non-small-cell lung cancer. N Engl J Med 2018;378:113-125.

9. Ramalingam SS, Vansteenkiste J, Planchard D, et al. Overall survival with osimertinib in untreated, EGFR-mutated advanced NSCLC. N Engl J Med 2020;382:41-50.

10. Nakagawa K, Garon EB, Seto T, et al. Ramucirumab plus erlotinib in patients with untreated, EGFR mutated, advanced non small cell lung cancer (RELAY): a randomised, double blind, placebo controlled, phase 3 trial. Lancet Oncol 2019;20:1655-1669.

11. Solomon BJ, Mok T, Kim D, et al. First-line crizotinib versus chemotherapy in ALK-positive lung cancer. N Engl J Med 2014;371:2167-2177.

12. Gadgeel SM, Gandhi L, Riely GJ, et al. Safety and activity of alectinib against systemic disease and brain metastases in patients with crizotinibresistant ALK-rearranged non-small-cell lung cancer (AF-002JG): results from the dose-finding portion of a phase 1/2 study. Lancet Oncol 2014;15: 1119-1128.
13. Peters $\mathrm{S}$, Camidge DR, Shaw AT, et al. Alectinib versus crizotinib in untreated ALK-positive non-small-cell lung cancer. N Engl J Med 2017;377: 829-838.

14. Camidge DR, Kim HR, Ahn M, et al. Brigatinib versus crizotinib in ALK-positive non-small-cell lung cancer. N Engl J Med 2018;379: 2027-2039.

15. Reck M, Rodriguez-Abreu D, Robinson AG, et al. Pembrolizumab versus chemotherapy for PD-L1-positive non-small-cell lung cancer. N Engl J Med 2016;375:1823-1833.

16. Reck M, Rodriguez-Abreu D, Robinson AG, et al. Updated analysis of KEYNOTE-024: pembrolizumab versus platinum-based chemotherapy for advanced non-small-cell lung cancer with PD-L1 tumor proportion score of 50\% or greater. J Clin Oncol 2019;37:537-546.

17. Mok TS, Wu Y, Kudaba I, et al. Pembrolizumab versus chemotherapy for previously untreated, PD-L1-expressing, locally advanced or metastatic non-small-cell lung cancer (KEYNOTE-042): a randomised, open-label, controlled, phase 3 trial. Lancet 2019;393:1819-1830.

18. Lisberg A, Cummings A, Goldman JW, et al. A phase II study of pembrolizumab in EGFR-mutant, PD-L1 +, tyrosine kinase inhibitor naïve patients with advanced NSCLC. J Thorac Oncol 2018;13:1138-1145

19. Gandhi L, Garassino MC. Pembrolizumab plus chemotherapy in lung cancer. N Engl J Med 2018;379:e18.

20. Socinski MA, Jotte RM, Cappuzzo F, et al. Atezolizumab for first-line treatment of metastatic nonsquamous NSCLC. N Engl J Med 2018;378: 2288-2301.

21. Paz-Ares L, Luft A, Vicente D, et al. Pembrolizumab plus chemotherapy for squamous non-small-cell lung cancer. N Engl J Med 2018;379: 2040-2051.

22. Jotte R, Cappuzzo F, Vynnychenko I, et al. Atezolizumab in combination with carboplatin and nab-paclitaxel in advanced squamous NSCLC (IMpower131): results from a randomized phase III trial [published online April 14, 2020]. J Thorac Oncol, doi: 10.1016/j.jtho.2020.03.028

23. Hellmann MD, Paz-Ares L, Caro RB, et al. Nivolumab plus ipilimumab in advanced non-small-cell lung cancer. N Engl J Med 2019;381:2020-2031.

24. Horn L, Mansfield AS, Szczesna A, et al. First-line atezolizumab plus chemotherapy in extensive-stage small-cell lung cancer. $\mathrm{N}$ Engl J Med 2018;379:2220-2229. 